\title{
Shared and distinct anatomical correlates of semantic and phonemic fluency revealed by lesion-symptom mapping in patients with ischemic stroke
}

\author{
J. Matthijs Biesbroek ${ }^{1}$ Martine J. E. van Zandvoort ${ }^{1,2}$ - L. Jaap Kappelle ${ }^{1}$. \\ Birgitta K. Velthuis ${ }^{3}$ - Geert Jan Biessels ${ }^{1}$ - Albert Postma ${ }^{1,2}$
}

Received: 5 December 2014/ Accepted: 17 March 2015/Published online: 5 May 2015

(c) The Author(s) 2015. This article is published with open access at Springerlink.com

\begin{abstract}
Semantic and phonemic fluency tasks are frequently used to test executive functioning, speed and attention, and access to the mental lexicon. In semantic fluency tasks, subjects are required to generate words belonging to a category (e.g., animals) within a limited time window, whereas in phonemic fluency tasks subjects have to generate words starting with a given letter. Anatomical correlates of semantic and phonemic fluency are currently assumed to overlap in left frontal structures, reflecting shared executive processes, and to be distinct in left temporal and right frontal structures, reflecting involvement of distinct memory processes and search strategies. Definite evidence for this assumption is lacking. To further establish the anatomical correlates of semantic and phonemic fluency, we applied assumption-free voxel-based and regionof-interest-based lesion-symptom mapping in 93 patients with ischemic stroke. Fluency was assessed by asking patients to name animals (semantic), and words starting with the letter $\mathrm{N}$ and $\mathrm{A}$ (phonemic). Our findings indicate that anatomical correlates of semantic and phonemic fluency
\end{abstract}

On behalf of the Utrecht VCI study group.

Electronic supplementary material The online version of this article (doi:10.1007/s00429-015-1033-8) contains supplementary material, which is available to authorized users.

J. Matthijs Biesbroek

j.m.biesbroek@umcutrecht.nl

1 Department of Neurology, Utrecht Stroke Center, Brain Center Rudolf Magnus, University Medical Center Utrecht, PO box 85500, G.03.232, 3508 GA Utrecht, The Netherlands

2 Experimental Psychology, Helmholtz Institute, Utrecht University, Utrecht, The Netherlands

3 Department of Radiology, University Medical Center Utrecht, Utrecht, The Netherlands overlap in the left inferior frontal gyrus and insula, reflecting shared underlying cognitive processes. Phonemic fluency additionally draws on the left rolandic operculum, which might reflect a search through phonological memory, and the middle frontal gyrus. Semantic fluency additionally draws on left medial temporal regions, probably reflecting a search through semantic memory, and the right inferior frontal gyrus, which might reflect the application of a visuospatial mental imagery strategy in semantic fluency. These findings establish shared and distinct anatomical correlates of semantic and phonemic fluency.

Keywords Phonemic - Semantic $\cdot$ Fluency $\cdot$ Lesionsymptom mapping - Anatomical correlates $\cdot$ Neural substrate

\section{Introduction}

Verbal fluency tasks, in which participants have to generate as many words as possible according to a specific criterion, are frequently used to test a circumscribed aspect of executive functioning that is referred to as energization (i.e., the voluntary generation of non-overlearned responses) (Robinson et al. 2012). Verbal fluency additionally depends on self-monitoring (which is also regarded as an executive process), processing speed and attention (van der Elst et al. 2005), working memory (Baldo et al. 2006; Robinson et al. 2012), and language processing including retrieval of appropriate responses from the mental lexicon (Juhasz et al. 2012). Verbal fluency is typically divided into two categories: (1) semantic fluency and (2) phonemic fluency (Lezak et al. 2004). In semantic fluency tests, participants are required to generate as many items belonging to a certain category (e.g., items in a grocery store, or animals 
in a zoo) as possible within a certain time window. In phonemic fluency tests, the subject is asked to generate as many words starting with a given letter as possible. Though both semantic and phonemic fluency tests assess energization and self-monitoring, they require different strategies for the creation and selection of appropriate novel responses, and depend on distinct memory processes (Baldo et al. 2006). Semantic fluency draws on semantic memory to retrieve previously obtained semantic knowledge on items belonging to a certain category, whereas in phonemic fluency appropriate items are selected based on phonological word knowledge (Baldo et al. 2006). Thus, semantic and phonemic fluency depend on partially shared (energization, self-monitoring, attention, processing speed, language) and partially distinct (search strategy, semantic versus phonological memory) cognitive processes.

Delineating the anatomical correlates of semantic and phonemic fluency by patient lesion-mapping techniques would improve our understanding of the overlap and differences in the cognitive processes involved and provide valuable insight in how and why certain neurological conditions hamper semantic or phonemic fluency in individual patients. There is substantial evidence from lesion studies for a crucial role of the left frontal lobe in both semantic and phonemic fluency (Robinson et al. 2012). Regarding the specific role of left frontal structures in semantic and phonemic fluency, fMRI studies in healthy subjects have consistently demonstrated distinct dorsalventral locations within the left inferior frontal gyrus for semantic and phonologic processes (Costafreda et al. 2006; Heim et al. 2008, 2009; Katzev et al. 2013). The role of the right frontal lobe in verbal fluency remains controversial: some studies reported impaired verbal fluency in a proportion of patients with right frontal lesions (Perret 1974; Martin et al. 1990; Loring et al. 1994; Robinson et al. 2012), but this is not confirmed by others (Milner 1964; Newcombe 1969). It is unclear whether the potential contribution of the right frontal lobe to verbal fluency is specific for either semantic or phonemic fluency, though findings of a recent lesion study suggest that right lateral frontal regions might be involved in semantic, but not phonemic fluency (Robinson et al. 2012). Moreover, a specific role of the left temporal lobe in verbal fluency has been demonstrated. Evidence from behavioral studies in healthy participants (Martin et al. 1994), functional imaging studies (Mummery et al. 1996; Gourovitch et al. 2000), and lesion studies (Henry and Crawford 2004; Baldo et al. 2006; Robinson et al. 2012) indicates that the temporal cortex underlies semantically based word retrieval but not phonemically driven word retrieval. Though the afore mentioned studies have provided important insights into the anatomical correlates of semantic and phonemic fluency, they have not resolved the issue entirely since (1)
fMRI studies in healthy subjects do not prove that activated structures are actually essential to the task, meaning that these findings should be confirmed with lesion studies to demonstrate a direct causal relation (Rorden and Karnath 2004), and (2) previously performed lesion studies have been limited by low spatial resolution or a strictly hypothesis-driven approach, thus ignoring potentially relevant brain regions. For example, the largest lesionsymptom study to date (67 patients) compared frontal lesions with posterior lesions, and additionally performed an analysis in which the frontal lobes were divided into three regions of interest (Robinson et al. 2012). Posterior lesions were associated with poor semantic fluency, but not phonemic fluency. However, due to the low spatial resolution (i.e., comparing frontal versus posterior), it remained unclear which posterior regions were crucially involved in semantic fluency. Another lesion-symptom mapping study performed voxel-wise analyses in 48 patients with left hemispheric stroke (Baldo et al. 2006). Left frontal lesions correlated with phonemic fluency, while left temporal lesions correlated with semantic fluency. A limitation of this study is that the impact of right hemispheric lesions on verbal fluency was not assessed (Baldo et al. 2006). The same limitation applies to a recent study of 31 patients with left hemispheric lesion in which an association was found between lesion in the left inferior fronto-occipital fasciculus and poor semantic, but not phonemic fluency (Almairac et al. 2014). In summary, there is a critical need for large-scale assumption-free patient lesion studies to further substantiate foregoing notions as well as resolve lingering controversies.

In the present study, we set out to further clarify the anatomical correlates of semantic and phonemic verbal fluency by applying hypothesis-free voxel-based (i.e., high spatial resolution) lesion-symptom mapping in a cohort of 93 patients with first-ever ischemic stroke. We expected that the anatomical correlates of semantic and phonemic fluency would overlap in left frontal regions and be discordant in left temporal and right frontal regions.

\section{Materials and methods}

\section{Subjects}

A flowchart of the inclusion of patients for the current study is provided in supplementary Fig. 1. Neuropsychological examination was performed in ischemic stroke patients who are admitted to our service in the setting of standard clinical care, if their condition permitted testing and testing facilities were available. All 243 ischemic stroke patients who were admitted from November 2005 through December 2012 and underwent neuropsychological assessment during admission 
were eligible for the present study (see supplementary Fig. 1). We subsequently applied a stepwise exclusion procedure to select patients without interfering pre-existent neurological conditions or brain lesions, in whom the ischemic lesion could be segmented on CT or MRI, and with available data on semantic and phonemic fluency (see supplementary Fig. 1). In the first step, we excluded 79 patients with pre-existent neurological conditions or imaging abnormalities: 19 patients with (probable) pre-existent cognitive impairment, 21 patients with prior stroke, 37 patients with old (silent) infarcts or severe white matter hyperintensity on brain imaging defined as Fazekas grade 3 [i.e., large confluent areas of white matter lesions (Fazekas et al. 1987)] on brain imaging, and 2 patients with recurrent stroke between brain imaging and neuropsychological examination. Cortical atrophy was not an exclusion criterion. In the second step, we excluded 43 patients for whom no brain imaging was available (no follow-up imaging after the acute admission scan in 24 patients, no ischemic lesion detected on follow-up imaging in 19 patients). In the final step, we excluded 28 patients who had no data on semantic and phonemic fluency. The application of these exclusion criteria resulted in the inclusion of 93 patients.

\section{Neuropsychological assessment}

Neuropsychological assessment was performed within one month after ischemic stroke (mean 7.5 days; range 1-30 days). We have previously demonstrated that the applied cognitive assessment battery is feasible and reliable in the acute stage (first days to weeks) of ischemic stroke (Nys et al. 2005b). Measures of fluency were obtained by asking patients to name as many words as possible (in the Dutch language) in the following categories: (1) animals, in $2 \mathrm{~min}$; (2) any word beginning with the letter N, in $1 \mathrm{~min}$; (3) any word beginning with the letter $\mathrm{A}$, in $1 \mathrm{~min}$. Educational level was divided into seven categories (scored according to Verhage 1964) with scores ranging from unfinished primary school education (category 1) to an academic degree (category 7) according to the Dutch educational system.

To investigate the relationship between semantic and phonemic fluency and measures of verbal and visuospatial memory and language, we additionally considered data on the Dutch version of the Rey Auditory Verbal Learning Test (RAVLT) (Rey 1958; Brand and Jolles 1985; van der Elst et al. 2005), the delayed Rey-Osterrieth Complex Figure Test (ROCF) (Osterrieth 1944), the Boston Naming Test (short form: 30 items) (Kaplan et al. 1983), and the Token Test (short form: 21 items) (De Renzi and Vignolo 1962). These tests were administered in the same session as the fluency tests. A detailed description of the administration of the RAVLT and ROCF is provided in the online supplementary methods.

\section{Generation of lesion maps}

The procedure for the generation of lesion maps has been previously described elsewhere (Biesbroek et al. 2014). Infarcts were manually segmented on either follow-up CT $(n=61)$, or MRI scans $(n=32)$. The infarct maps were registered to the T1 MNI-152 (Montreal Neurological Institute) template utilizing a lesion-masking approach (Brett et al. 2001; Fonov et al. 2009). Registration of MRI images was performed using elastix; CT images were registered using an in-house developed algorithm which is described elsewhere (Klein et al. 2010; Kuijf et al. 2013). A detailed description of the generation, registration and quality checks of the lesion maps is provided in the online supplementary methods.

\section{Statistics}

Phonemic fluency was defined as the sum of correct, nonrepeated words that an individual produced in the $\mathrm{N}$ and $\mathrm{A}$ letter trials. Semantic fluency was defined as the total number of correct, non-repeated animals that an individual named in the animal naming trial. Measures of phonemic and semantic fluency, and performance on the RAVLT, delayed ROCF copy test, Boston Naming Test, and Token Test were transformed to $z$ scores and corrected for age, sex and level of education for each individual patient using linear regression (i.e., based on the group means and standard deviations). Pearson correlations were used to compute the correlation between semantic and phonemic fluency and measures of verbal and spatial memory. To assess the prevalence of aphasia in our study cohort, performance on the Boston Naming Test and the Token Test was dichotomized using previously described norms; performance below the 5th percentile was considered abnormal (Heesbeen 2002).

Rather than focusing on specific brain regions, patients with lesions anywhere in the brain were included. We first performed assumption-free VLSM to assess the association between the presence of a lesion and semantic and phonemic fluency in a given voxel (Rorden and Karnath 2004; Kimberg et al. 2007; Rorden et al. 2007), and complemented these voxel-wise analyses with a region-ofinterest-based approach. VLSM analyses were done using Non-Parametric Mapping (most recent version, December 2012; settings: $t$ test, univariate analysis) (Rorden et al. 2007). The Non-Parametric Mapping software provides two tests for VLSM: the parametric $t$ test and the nonparametric Brunner-Munzel (BM) statistic. Because the $t$ test has higher power than the BM statistic in small sample sizes, and because the $t$ test is particularly robust as it becomes conservative rather than liberal (i.e., reporting false alarms) when the underlying assumptions are 
violated, we chose to use the $t$ test in our main analyses (Rorden et al. 2007). Voxels affected by ischemic lesions in less than 3 patients were not considered for analysis. Correction for multiple testing was performed using a false discovery rate threshold (FDR) with $q<0.05$. To assess the robustness of the VLSM results, we additionally performed a qualitative lesion subtraction analysis using dichotomized measures of fluency as the dependent variable (i.e., abnormal versus normal), instead of using $z$ scores. Phonemic and semantic fluency were dichotomized using previously described norms that were obtained in a cohort of healthy Dutch individuals; performance below the 5th percentile was considered abnormal (Deelman et al. 1981; Brand et al. 2007; Nys et al. 2005a). Because dichotomization of performance results in a decrease in statistical power and does not account for severity of the deficit, we chose to use the continuous outcome (analyzed with $t$ test) in our main analyses.

In the next step, we complemented the voxel-based analyses with a region-of-interest-based analysis to quantify the impact of regional lesion volumes on phonemic and semantic fluency. The regions of interest were selected based on the VLSM results. For this purpose, regions of interest for 90 cerebral cortical regions were extracted from the automatic anatomical labeling (AAL) atlas (TzourioMazoyer et al. 2002). These 90 regions were projected on the VLSM results and the amount of voxels with a statistically significant association within each region was assessed quantitatively. Regions that appeared to be involved in phonemic or semantic fluency (operationally defined as at least 100 significant voxels within a specific region) were selected for the region-of-interest-based analyses visually. Next, infarct volume within the selected regions was calculated for every patient. These regional infarct volumes were entered as independent variables in a linear regression model with phonemic and semantic fluency as the dependent variables, before and after adding total infarct volume to the model. The rationale behind adding infarct volume as a covariate was that brain regions that are crucial when performing a certain task should predict performance, independent of total infarct volume. However, it should be kept in mind that adding infarct volume as a covariate will decrease statistical power, especially when relevant anatomical structures correlate with large infarcts (due to the anatomy of the cerebral arteries) (Karnath et al. 2004). For this reason, VLSM analyses are often not corrected for total infarct volume (Baldo et al. 2006; Haramati et al. 2008; Molenberghs and Sale 2011; Vossel et al. 2011; Fridriksson et al. 2013; Magnusdottir et al. 2013). Lesion studies in which a correction for total infarct volume is applied generally show VLSM results that are not corrected for multiple testing to compensate for reduced statistical power (Karnath et al. 2004;
Schwartz et al. 2009). Instead, we chose to apply the correction for total infarct volume in the region-of-interestbased analyses because these analyses do not require correction for multiple testing (similar to Thothathiri et al. 2012; Biesbroek et al. 2014).

\section{Results}

Clinical characteristics of the study cohort are provided in Table 1. Eighteen out of 93 patients had impaired semantic fluency (19\%); 29 (31\%) patients had impaired phonemic fluency. Thirteen patients had both impaired semantic and phonemic fluency. Impaired semantic and phonemic fluency was most prevalent in patients with left hemispheric lesions, but was also present in a substantial number of patients with right hemispheric lesions (Table 2). Semantic and phonemic fluency were significantly correlated $(r=0.642 ; p<0.001)$. Both semantic and phonemic fluency were correlated with measures of verbal memory (working memory, delayed recall, recognition memory) and language (Boston Naming Test, Token Test) (Table 3). In contrast, semantic fluency was correlated with visuospatial memory performance $(r=0.233 ; p=0.032)$, whereas phonemic fluency was not $(r=0.084$; $p=0.444)$. Thirteen out of 85 patients with data on the Token Test $(15 \%)$ and 34 out of 91 patients with data on the Boston Naming Test (37 \%) had impaired performance on these language tests.

Table 1 Characteristics of the study cohort

\begin{tabular}{ll}
\hline Characteristics & $\begin{array}{l}\text { Study cohort } \\
(n=93)\end{array}$ \\
\hline Demographic characteristics & $59.5(14.9)$ \\
Age, mean (SD) & $53(57)$ \\
Male, $n(\%)$ & $5(2-7)$ \\
Education, median (range) & \\
Hand preference, $n(\%)^{\mathrm{b}}$ & $82(89)$ \\
Right & $9(10)$ \\
Left & $1(1)$ \\
Ambidexter & \\
Neuropsychological examination & $7.5(5.1 ; 1-30)$ \\
Time interval between stroke and NPE in days, \\
$\quad$ mean (SD; range) & $8.0(4.3 ; 0-22)$ \\
No. words letter A (1 min) & $7.7(4.4 ; 0-20)$ \\
No. words letter N (1 min) & $23.1(10.4 ;$ \\
No. animals (2 min) & $0-51)$ \\
& \\
\hline$N P E$ neuropsychological examination & \\
a Education scored according to Verhage scoring system (scale 1-7) \\
b Data on hand preference missing in one patient
\end{tabular}


Table 2 Location of ischemic lesion in relation to the presence of impaired semantic or phonemic fluency

\begin{tabular}{llllll}
\hline Lesion location, $n(\%)$ & \multicolumn{2}{l}{ Impaired semantic fluency } & & \multicolumn{2}{l}{ Impaired phonemic fluency } \\
\cline { 2 - 3 } & Yes $(n=18)$ & No $(n=75)$ & & Yes $(n=29)$ & No $(n=64)$ \\
\hline Left hemisphere $(n=34)$ & $11(61 \%)$ & $23(31 \%)$ & & $16(55 \%)$ & $18(28 \%)$ \\
Right hemisphere $(n=40)$ & $5(28 \%)$ & $35(47 \%)$ & & $8(28 \%)$ & $32(50 \%)$ \\
Infratentorial $(n=12)$ & $1(6 \%)$ & $11(15 \%)$ & & $2(7 \%)$ & $10(16 \%)$ \\
Multiple locations $(n=7)$ & $1(6 \%)$ & $6(8 \%)$ & & $3(10 \%)$ & $4(6 \%)$ \\
\hline
\end{tabular}

\begin{tabular}{lll}
\hline & Phonemic fluency $(\mathrm{N}+\mathrm{A})$ & Semantic fluency (animal) \\
\hline Semantic fluency & $0.642(p<0.001)$ & - \\
${\text { RAVLT total recall trial } 1-5^{\mathrm{a}}}^{\mathrm{R}}$ & $0.558(p<0.001)$ & $0.583(p<0.001)$ \\
RAVLT recollection $^{\mathrm{a}}$ & $0.493(p<0.001)$ & $0.517(p<0.001)$ \\
RAVLT recognition $^{\mathrm{a}}$ & $0.425(p<0.001)$ & $0.566(p<0.001)$ \\
Delayed ROCF $^{\mathrm{b}}$ & $0.084(p=0.444)$ & $0.233(p=0.032)$ \\
Boston Naming Test $^{\mathrm{c}}$ & $0.405(p<0.001)$ & $0.571(p<0.001)$ \\
Token Test $^{\mathrm{d}}$ & $0.557(p<0.001)$ & $0.582(p<0.001)$ \\
\hline
\end{tabular}

The presented $p$ values correspond with a two-tailed test

RAVLT Rey Auditory Verbal Learning Test, ROCF Rey-Osterrieth Complex Figure Test

${ }^{\text {a }}$ Based on 89 patients with data on the RAVLT

b Based on 85 patients with data on delayed ROCF

c Based on 91 patients with data on Boston Naming Test

${ }^{d}$ Based on 85 patients with data on Token Test

\section{Voxel-based lesion-symptom mapping}

The spatial distribution of infarcts is illustrated by the lesion prevalence map in Fig. 1. Lesion prevalence was highest for voxels in the right cerebral hemisphere in the vascular territory of the middle cerebral artery. VLSM identified large overlapping anatomical correlates for semantic and phonemic fluency in the left frontal lobe (inferior and medial frontal, and precentral gyri, and rolandic operculum, insula, and putamen). Anatomical correlates were discordant in the following regions: lesions in the left medial temporal lobe (hippocampus, and perihippocampal, inferior temporal, lingual, and fusiform gyri) and right frontal lobe (inferior frontal gyrus and periventricular white matter) were associated with poor semantic, but not phonemic fluency. In contrast, lesions in the left middle frontal gyrus were associated with poor phonemic, but not semantic fluency. The VLSM results for semantic and phonemic fluency are provided in Fig. 2. The number of significant voxels for each region is provided in Table 4 . To assess the robustness of the VLSM results, we additionally performed a qualitative lesion subtraction analysis using dichotomized cognitive performance as the dependent variable, instead of using $z$ scores. The results of these lesion subtraction analyses were essentially the same as the VLSM results (Fig. 3).

\section{Region-of-interest-based analyses}

Next, we analyzed the impact of lesion volumes within specific cortical regions of interest on semantic and phonemic fluency (Table 5). Infarct volume within the left inferior frontal gyrus and left insula inversely correlated with both semantic and phonemic fluency. Infarct volume within the left rolandic operculum and left medial frontal gyrus inversely correlated with phonemic, but not semantic fluency; in contrast, infarct volume within the left putamen inversely correlated with semantic, but not phonemic fluency. The discordance of anatomical correlates in the left medial temporal lobe and right frontal lobe was reproduced: there was a statistically significant inverse correlation between infarct volume within the left hippocampus, perihippocampal, inferior temporal, lingual, and fusiform gyri, and the right inferior frontal gyrus, and semantic fluency, but not phonemic fluency. The impact of regional infarct volume on semantic fluency was greatest in the left parahippocampal gyrus (unstandardized coefficient (B) -0.45 ; $95 \%$ CI -0.75 to -0.15 ); the impact of regional infarct volume on phonemic fluency was greatest in the left rolandic operculum $(B-0.44 ; 95 \% \mathrm{CI}-0.70$ to $-0.17)$ ). The results of the linear regression analyses remained essentially the same after additional adjustment for total infarct volume (supplementary Table 1). 


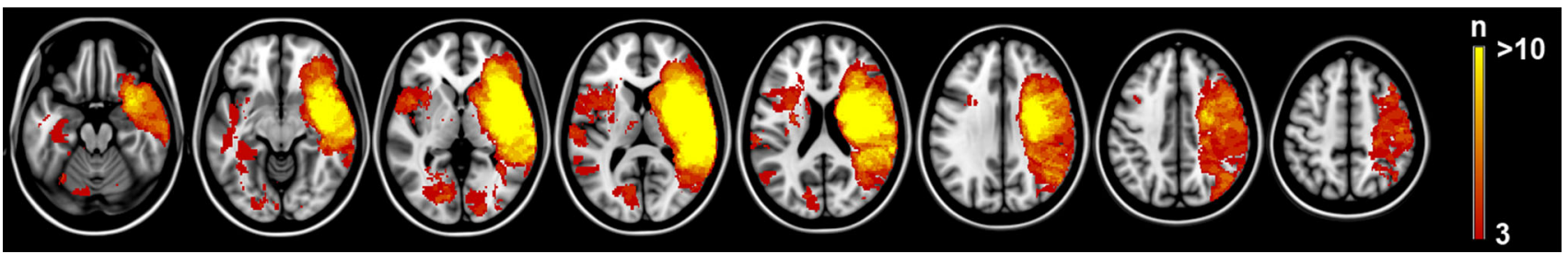

Fig. 1 Distribution of ischemic lesions. Voxels that are damaged in at least three patients are projected on the $1 \mathrm{~mm}$ MNI-152 template ( $Z$ coordinates: $-20,-10,0,10,20,30,40,50)$. Bar the number of patients with a lesion for each voxel. The right hemisphere is depicted on the right

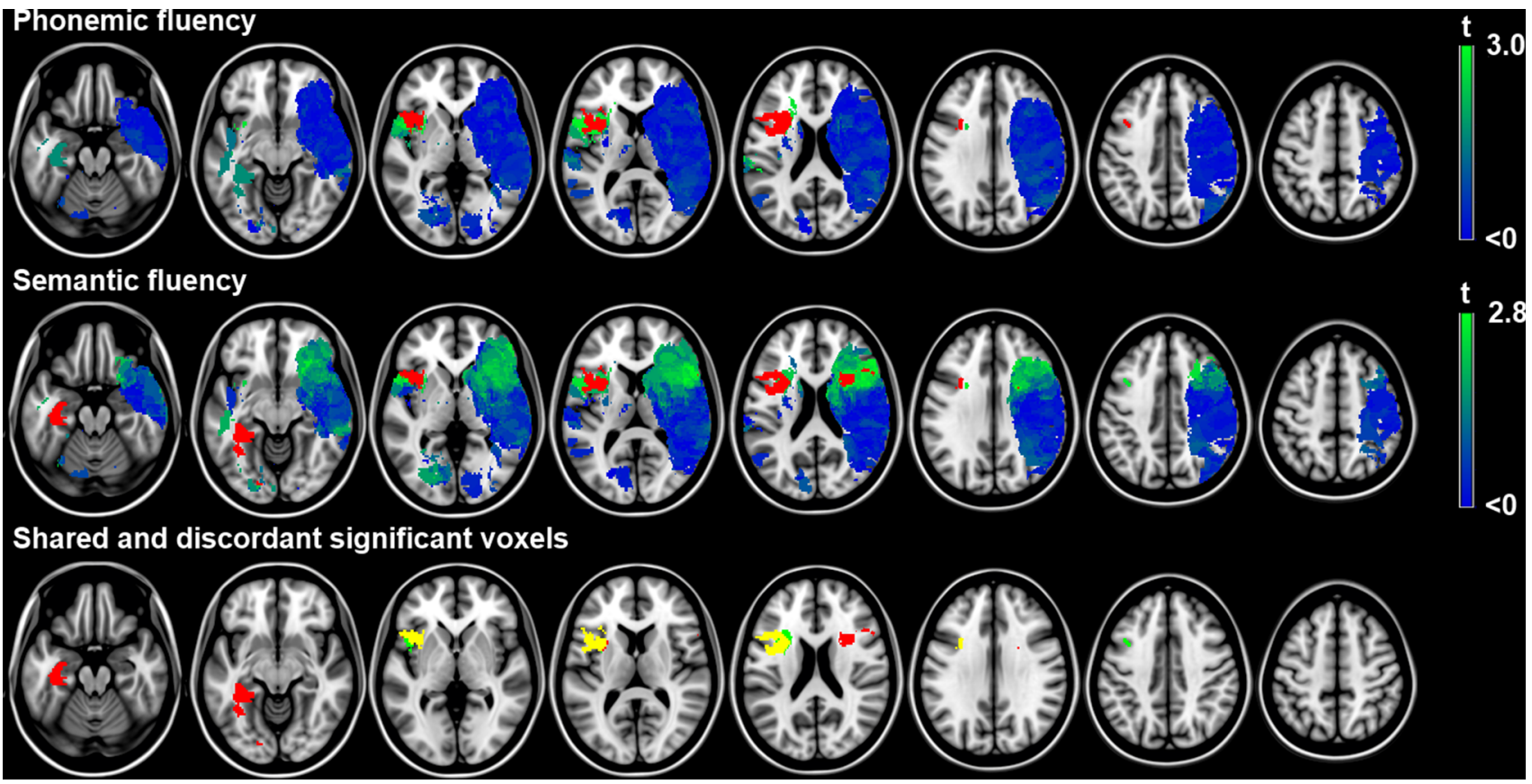

Fig. 2 Voxel-based lesion-symptom mapping results. Map of the voxel-wise association ( $t$ statistic) between the presence of a lesion and cognitive performance. Voxels exceeding the false discovery rate threshold $(q=0.05)$ are rendered in red. Non-significant voxels are rendered on a scale from blue $(t<0)$ to bright green ( $t$ value just below threshold). Negative $t$ values (meaning the presence of a lesion was correlated with better cognitive performance) were not statistically significant. Lower row voxels with a statistically significant

\section{Discussion}

The findings of the current study indicate that semantic and phonemic fluency have partially shared and partially distinct neural underpinnings. Anatomical correlates overlap in the left inferior frontal gyrus and insula, reflecting shared underlying cognitive processes. Phonemic fluency additionally draws on the left rolandic operculum and the left middle frontal gyrus. In contrast, left medial temporal regions and the right inferior frontal gyrus are crucially involved in semantic, but not phonemic fluency.

The main strengths of the current study are the substantial sample size, the assumption-free nature of the inverse association with performance on either semantic fluency (red), phonemic fluency (green) or both (yellow) are depicted. Note that the anatomical correlates overlap in left frontal regions, but are discordant in left temporal and right frontal regions. Semantic and phonemic fluency were corrected for age, sex and level of education using linear regression. The results are projected on the MNI 1-mm template $(Z$ coordinates: $-20,-10,0,10,20,30,40,50)$. The right hemisphere is depicted on the right

analyses (as opposed to hypothesis-driven analyses, in which the analyses are focused on predefined regions of interest), and the application of quantitative voxel-wise analyses that provides good spatial resolution. Our findings regarding the crucial role of left frontal structures in both semantic and phonemic fluency and involvement of the left temporal lobe in semantic, but not phonemic fluency are in line with previous findings (Martin et al. 1994; Mummery et al. 1996; Gourovitch et al. 2000; Henry and Crawford 2004; Baldo et al. 2006; Robinson et al. 2012). However, these previous studies focused on the left hemisphere, or compared posterior/temporal lesions in either hemisphere with frontal regions. As such, the current study is the first 
Table 4 Voxel-based lesion-symptom mapping results: tested and significant voxels for each region of interest

\begin{tabular}{lccccc}
\hline $\begin{array}{l}\text { Anatomical regions } \\
\text { (AAL atlas) }\end{array}$ & $\begin{array}{l}\text { Patients with } \\
\text { lesion }(n)^{*}\end{array}$ & $\begin{array}{l}\text { Region size, } \\
\text { voxels }(n)\end{array}$ & $\begin{array}{l}\text { Tested } \\
\text { voxels }(n)\end{array}$ & $\begin{array}{l}\text { Significant voxels } \\
\text { semantic }[n(\%)]\end{array}$ & $\begin{array}{l}\text { Significant voxels } \\
\text { phonemic }[n(\%)]\end{array}$ \\
\hline Middle frontal gyrus L & 7 & 38,722 & 111 & $7(6.3)$ & $111(100)$ \\
Inferior frontal gyrus operc L & 8 & 8271 & 2926 & $1776(60.7)$ & $1832(62.6)$ \\
Inferior frontal gyrus triang L & 8 & 20,104 & 593 & $559(94.3)$ & $581(98.0)$ \\
Rolandic operculum L & 11 & 7939 & 2670 & $682(25.5)$ & $317(26.9)$ \\
Insula L & 17 & 15,025 & 5314 & $3158(59.4)$ & $340(44.4)$ \\
Precentral gyrus L & 13 & 28,174 & 766 & $383(22.6)$ & $297(17.5)$ \\
Putamen L & 17 & 7942 & 1695 & $774(88.6)$ & 0 \\
Hippocampus L & 8 & 7469 & 874 & $839(99.5)$ & 0 \\
Parahippocampal gyrus L & 4 & 7891 & 843 & $2615(67.5)$ & 0 \\
Fusiform gyrus L & 8 & 18,333 & 3874 & $329(59.8)$ & 0 \\
Inferior temporal gyrus L & 7 & 25,647 & 550 & $156(3.4)$ & 0 \\
Lingual gyrus L & 12 & 16,932 & 4543 & $107(1.1)$ & 0 \\
Inferior frontal gyrus operc R & 27 & 11,174 & 9340 & $158(1.4)$ & \\
Inferior frontal gyrus triang R & 20 & 17,132 & 11,470 & \\
\hline Regions & \\
\hline
\end{tabular}

Regions that appeared to be involved in semantic or phonemic fluency are shown (definition: significant association between lesion and performance in at least 100 voxels). The remaining 76 regions contained $<100$ significant voxels for both semantic and phonemic fluency; these regions are not shown here

$R$ right, $L$ left

* How many of the 93 included patients had a lesion that overlapped ( $\geq 1$ voxel) with the specified region of interest

to determine the anatomical correlates of semantic and phonemic fluency in an assumption-free, voxel-wise manner, taking into account lesions in both hemispheres. Furthermore, our findings provide new insights in the involvement of the right frontal lobe in verbal fluency: lesions in the right inferior frontal gyrus and periventricular frontal white matter are associated with poor semantic, but not phonemic fluency. Thus, right dorsolateral frontal structures are involved in semantic, but not phonemic fluency.

The observed partially shared and partially discordant anatomical correlates of semantic fluency reflect the involvement of multi-component cognitive processes. The shared correlates in the left inferior frontal gyrus and insula are likely to reflect word production and processing. This is further underlined by the strong correlations between both semantic and phonemic fluency, and the Boston Naming Test and Token Test. The observed involvement of the left rolandic operculum in phonemic fluency, but not in semantic fluency might reflect a search through phonological memory. In contrast, involvement of left medial structures in semantic fluency might reflect a search through semantic memory. Indeed, a crucial role of left medial temporal structures in verbal semantic memory has been clearly established (Tulving and Markowitsch 1998; Levy et al. 2004; Binder et al. 2009; Groussard et al. 2010), while perisylvian regions (including the rolandic operculum) are known to be involved in accessing phonological representations and phoneme selection and production (Alexander and Hillis 2008).

To our knowledge, there is currently no well-established theory that would explain why right dorsolateral frontal structures are involved in semantic, but not phonemic fluency. We speculate here that the differential involvement of right frontal regions might reflect a 'visuospatial mental imagery strategy', in which the subject generates mental images of appropriate items (animals in our case). Such a strategy could be helpful when searching through semantic memory, but would not be appropriate when searching through phonological memory. The application of a strategy involving mental imagery of concrete things in semantic fluency tasks has been previously suggested, based on the observation that patients often report imagining themselves walking through a zoo or a farm when asked to name as many animals as possible (Baldo et al. 2006). The right dorsolateral prefrontal cortex is known to be involved in keeping spatial information 'on-line' and in strategy formation (Miotto et al. 1996; van Asselen et al. 2006). Thus, the application of a visuospatial mental imagery strategy in semantic fluency would likely depend on right dorsolateral frontal regions. Furthermore, the observed correlation of performance on the visuospatial memory test with semantic fluency, but not with phonemic fluency (Table 3), would fit with the application of a visuospatial mental imagery strategy in semantic fluency. The process of mental imagery of animals in semantic fluency tasks can 

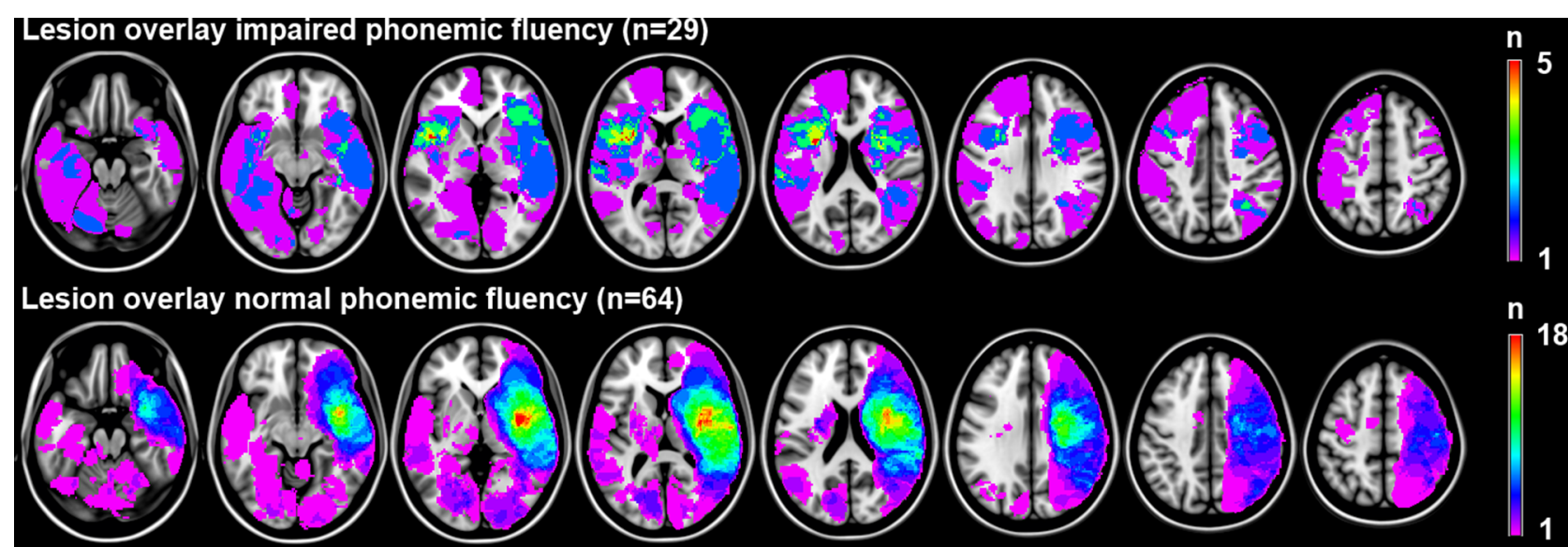

Lesion subtraction phonemic fluency
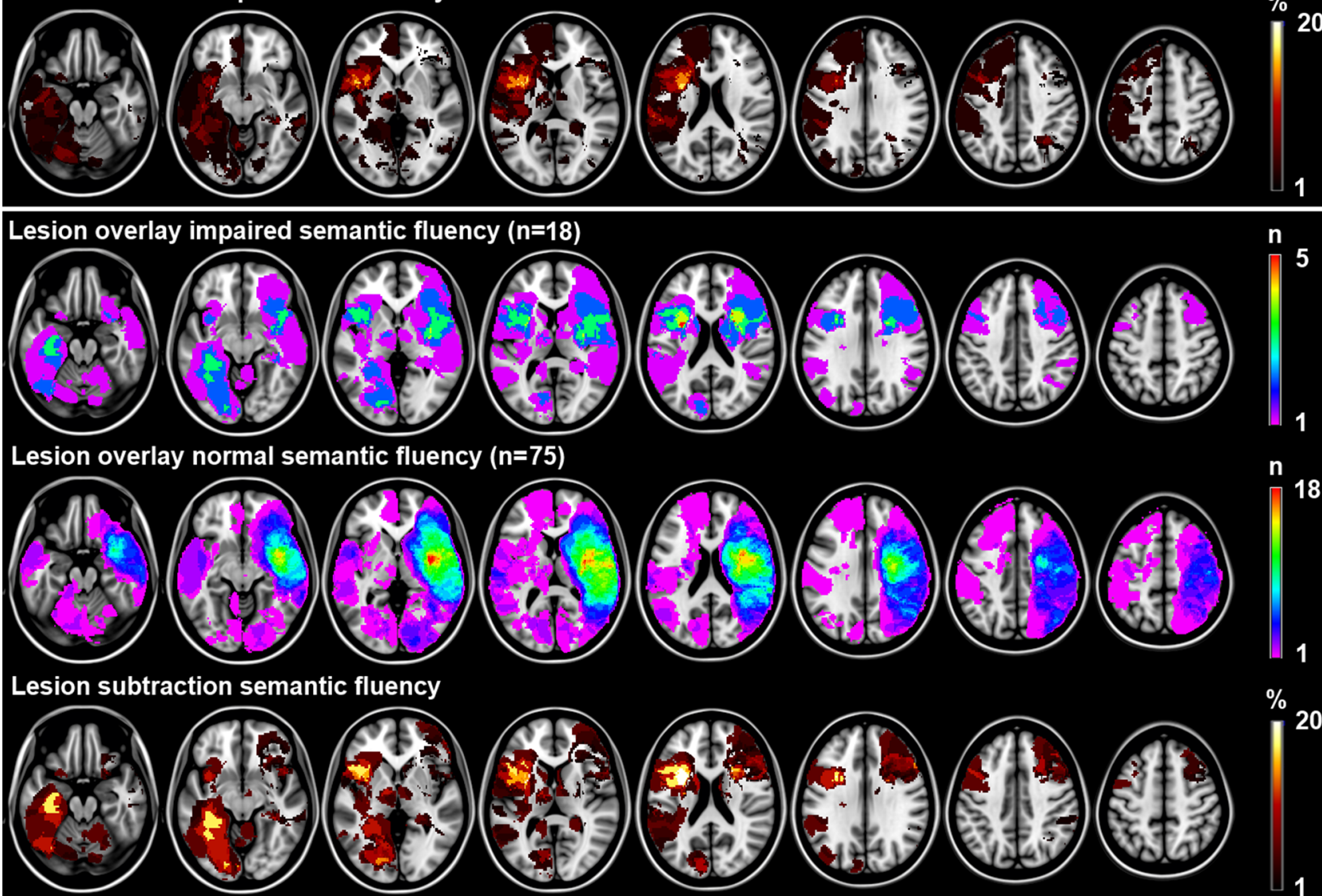

$\%$

Fig. 3 Lesion subtraction analyses with dichotomized fluency measures as outcome. Lesion overlay and subtraction plots of dichotomized measures of fluency (impaired yes/no based on previously described norms). The overlay plots show the number of patients with a lesion for a given voxel separately for patients with impaired and normal performance. The lesion subtraction plots show which voxels are more frequently affected in patients with impaired performance compared to patients with normal performance. For example, the semantic fluency overlay plots show that 3 out of 18 patients $(17 \%)$ with impaired semantic fluency have a lesion in the left hippocampus,

perhaps be compared to the imagery that is needed for design fluency tasks, which would fit with previous findings that right lateral frontal lesions result in impaired whereas none of the $75(0 \%)$ patients with normal semantic fluency have a lesion in the left hippocampus. The lesion subtraction plot shows the resulting $17 \%$ difference in lesion prevalence. This finding suggests a crucial role of the left hippocampus in semantic fluency. The lesion subtraction and voxel-based lesion-symptom mapping results are essentially the same: phonemic and semantic fluency both depend on left frontal structures. Semantic fluency additionally depends on left medial temporal and right frontal structures, whereas phonemic fluency does not. The right hemisphere is depicted on the right

design fluency (Robinson et al. 2012). Unfortunately, we have no data on (visuospatial) strategy formation to further substantiate this hypothesis. Further studies are needed to 


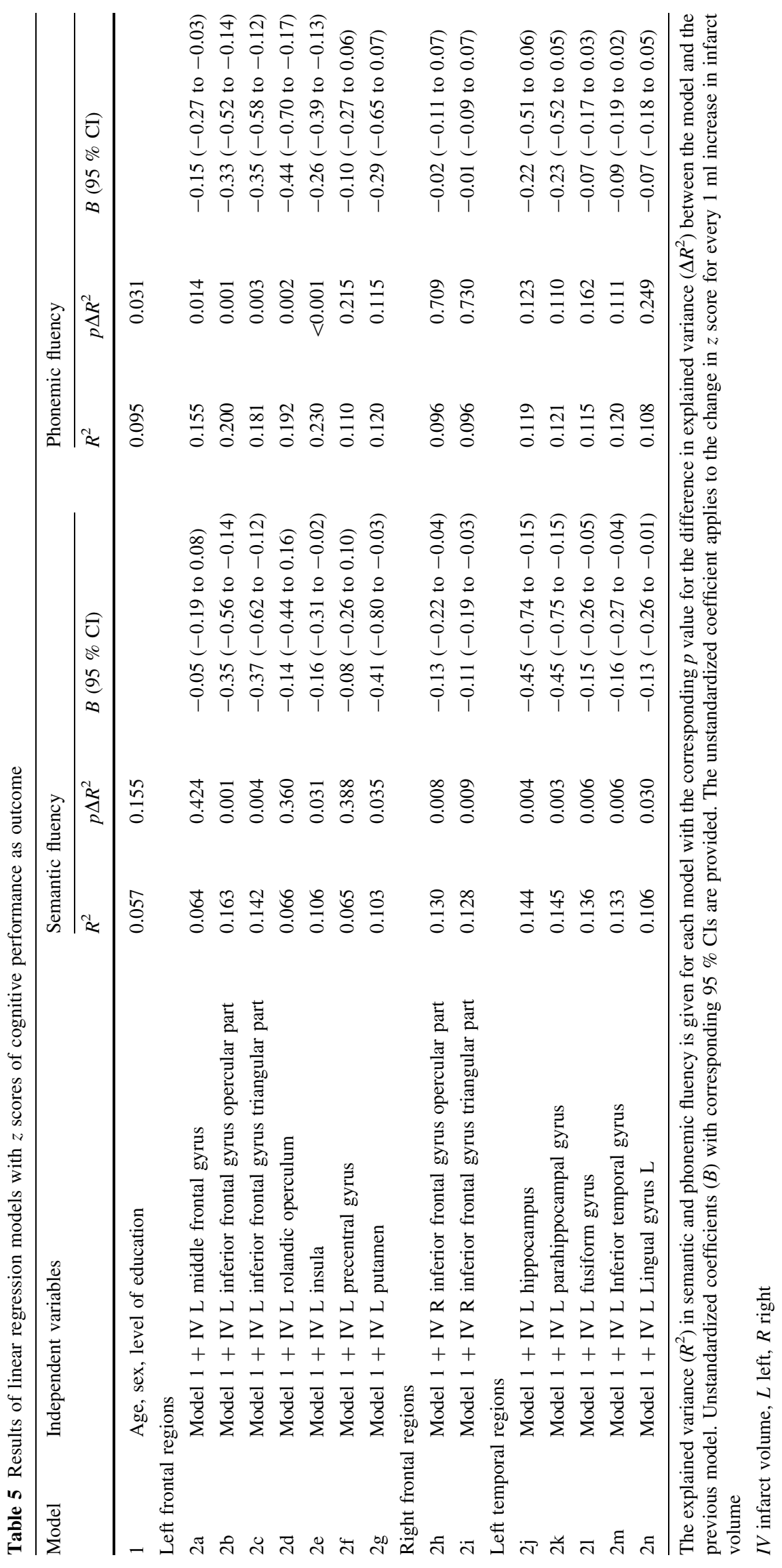


determine whether the contribution of right dorsolateral frontal structures indeed reflects the application of a visuospatial mental imagery strategy in semantic fluency.

A potential limitation of the current study is the relatively low lesion frequency in the left cerebral hemisphere in the voxel-based analyses (despite the substantial number of patients with left hemispheric lesions). The reason for this lies in the fact that neuropsychological examination is not always feasible in patients with severe global aphasia, especially when applying tests that require processing of verbal information. The decision whether or not to perform a neuropsychological examination in patients with global aphasia was made by the treating clinical neuropsychologist as these tests were always performed in the setting of standard clinical care. The presence of aphasia was not an exclusion criterion for the current study. Despite the relatively low lesion frequency in left hemispheric voxels, we were able to demonstrate differential involvement of left temporal and left frontal regions in semantic and phonemic fluency. Second, we used both CT and MRI scans for lesion segmentation, which is not uncommon in lesion-symptom mapping studies in stroke (Karnath et al. 2004; Schwartz et al. 2009; Thothathiri et al. 2012; Robinson et al. 2012; Theys et al. 2013). Both modalities allow for accurate detection of the location on the ischemic lesion. However, the boundary of the lesion might be drawn differently between modalities. In addition, this boundary is also influenced by the elapsed time between stroke onset and CT/MRI scan acquisition. The variability in lesion segmentation could be minimized by applying a single scan modality in a certain time window (e.g., MRI acquired 48-72 h after stroke onset). However, we chose for a robust design including as many patients as possible (with either CT or MRI scans) to optimize statistical power, while accepting some heterogeneity in scan acquisition (Biesbroek et al. 2014). It should be noted that the marked differences in anatomical correlates of discriminability in the left temporal and right frontal lobe cannot be attributed to slight variability in the segmentation of lesion boundaries. The level of difficulty of fluency tests may differ per letter (for phonological fluency) and category (for semantic fluency) and depends on task duration as well (i.e., 1 versus $2 \mathrm{~min}$ ). The level of difficulty of the test could affect the function-structure mapping because an increased level of difficulty might theoretically result in recruitment of nonspecific brain regions (Dräger et al. 2004). However, this cannot explain the observed association between lesions in right frontal and left temporal regions and poor semantic, but not phonemic fluency, because the semantic fluency test was in fact less difficult than the phonemic fluency tests. Patients on average named more animals (mean of 23 in $2 \mathrm{~min}$ ) than words starting with the letter $\mathrm{N}$ and $\mathrm{A}$ combined (mean of 16 words in
2 min); see Table 1. Furthermore, we did not directly compare performance on both tests (which would be problematic because of the assumed differences in difficulty). Instead, performance on each test was transformed to $z$ scores based on individual variation in test performance. We subsequently identified the anatomical correlates of each task separately, followed by a qualitative comparison to identify shared and unique anatomical correlates.

In conclusion, our findings indicate that both semantic and phonemic fluency depend on left frontal structures, while left medial temporal and right dorsolateral frontal structures are involved in semantic, but not phonemic fluency. The involvement of left medial temporal regions in semantic fluency most likely reflects retrieval of appropriate responses from semantic memory. Phonemic fluency depends more strongly on left perisylvian regions which might reflect retrieval of responses from phonological memory. The involvement of right dorsolateral frontal regions in semantic, but not phonemic, might reflect the application of a spatial strategy.

Acknowledgments We gratefully acknowledge Nick A. Weaver, Hugo J. Kuijf, and Pieter C. Vos for their help in generating the lesion maps. Furthermore, we would like to acknowledge Nathan Van der Stoep, Haike van Stralen, Linda Schoo, and Sophie Heringa for performing the neuropsychological examinations, and the members of the Vascular Cognitive Impairment Study group of the University Medical Center Utrecht. The work of J. M. Biesbroek was supported by a Neuroscience \& Cognition grant from Utrecht University. The work of G. J. Biessels was supported by VIDI grant 91711384 from ZonMw, The Netherlands Organisation for Health Research and Development, and by grant 2010T073 from the Netherlands Heart Foundation. The funders had no role in study design, data collection and analysis, decision to publish, or preparation of the manuscript.

Conflict of interest The authors declare that they have no conflict of interest.

Open Access This article is distributed under the terms of the Creative Commons Attribution License which permits any use, distribution, and reproduction in any medium, provided the original author(s) and the source are credited.

\section{References}

Alexander MP, Hillis AE (2008) Aphasia. In: Goldenberg G, Miller B (eds) Handbook of clinical neurology. Elsevier Press, Amsterdam, p 300

Almairac F, Herbet G, Moritz-Gasser S, de Champfleur NM, Duffau $\mathrm{H}$ (2014) The left inferior fronto-occipital fasciculus subserves language semantics: a multilevel lesion study. Brain Struct Funct. doi:10.1007/s00429-014-0773-1

Baldo JV, Schwartz S, Wilkins D, Dronkers NF (2006) Role of frontal versus temporal cortex in verbal fluency as revealed by voxelbased lesion-symptom mapping. J Int Neuropsychol Soc 12:896-900 
Biesbroek JM, van Zandvoort MJ, Kuijf HJ, Weaver NA, Kappelle LJ, Vos PC et al (2014) The anatomy of visuospatial construction revealed by lesion-symptom mapping. Neuropsychologia 62:68-76

Binder JR, Desai RH, Graves WW, Conant LL (2009) Where is the semantic system? A critical review and meta-analysis of 120 functional neuroimaging studies. Cereb Cortex 19:2767-2796

Brand N, Jolles J (1985) Learning and retrieval rate of words presented auditorily and visually. J Gen Psychol 112:201-210

Brand AM, van den Berg E, Manschot SM, Biessels GJ, Kappelle LJ, de Haan EH et al (2007) A detailed profile of cognitive dysfunction and its relation to psychological distress in patients with type 2 diabetes mellitus. J Int Neuropsychol Soc 13:288-297

Brett M, Leff AP, Rorden C, Ashburner J (2001) Spatial normalization of brain images with focal lesions using cost function masking. Neuroimage 14:486-500

Costafreda SG, Fu CH, Lee L, Everitt B, Brammer MJ, David AS (2006) A systematic review and quantitative appraisal of fMRI studies of verbal fluency: role of the left inferior frontal gyrus. Hum Brain Mapp 27:799-810

De Renzi E, Vignolo LA (1962) The token test: a sensitive test to detect receptive disturbances in aphasics. Brain 85:665-678

Deelman BG, Koning-Haanstra M, Liebrand WBG (1981) SAN-test: een afasie test voor auditief en mondeling taalgebruik. Swets \& Zeitlinger, Lisse

Dräger B, Jansen A, Bruchmann S, Förster AF, Pleger B, Zwitserlood P, Knecht S (2004) How does the brain accommodate to increased task difficulty in word finding? A functional MRI study. Neuroimage 23:1152-1160

Fazekas F, Chawluk JB, Alavi A, Hurtig HI, Zimmerman RA (1987) MR signal abnormalities at $1.5 \mathrm{~T}$ in Alzheimer's dementia and normal aging. AJR Am J Roentgenol 149:351-356

Fonov V, Evans A, McKinstry R, Almli C, Collins D (2009) Unbiased nonlinear average age-appropriate brain templates from birth to adulthood. Neuroimage 47:S102

Fridriksson J, Guo D, Fillmore P, Holland A, Rorden C (2013) Damage to the anterior arcuate fasciculus predicts non-fluent speech production in aphasia. Brain 136:3451-3460

Gourovitch ML, Kirkby BS, Goldberg TE, Weinberger DR, Gold JM, Esposito $\mathrm{G}$ et al (2000) A comparison of rCBF patterns during letter and semantic fluency. Neuropsychology 14:353-360

Groussard M, Viader F, Hubert V, Landeau B, Abbas A, Desgranges $B$ et al (2010) Musical en verbal semantic memory: two distinct neural networks? Neuroimage 49:2764-2773

Haramati S, Soroker N, Dudai Y, Levy DA (2008) The posterior parietal cortex in recognition memory: a neuropsychological study. Neuropsychologia 46:1756-1766

Heesbeen IME (2002) Diagnostiek en herstelmeting van taalproblemen na niet-aangeboren hersenletsel. Dissertation, Utrecht University

Heim S, Eickhoff SB, Amunts K (2008) Specialisation in Broca's region for semantic, phonological, and syntactic fluency? Neuroimage 15:1362-1368

Heim S, Eickhoff SB, Amunts K (2009) Different roles of cytoarchitectonic BA 44 and BA 45 in phonological and semantic verbal fluency as revealed by dynamic caudal modelling. Neuroimage 48:616-624

Henry JD, Crawford JR (2004) A meta-analytic review of verbal fluency performance following focal cortical lesions. Neuropsychologia 18:284-295

Juhasz BG, Chambers D, Shesler LW, Haber A, Kurtz MM (2012) Evaluating lexical characteristics of verbal fluency output in schizophrenia. Psychiatry Res 200:177-183

Kaplan EF, Goodglass H, Weintraub S (1983) The Boston naming test (2nd edition). Lea \& Febiger, Philadelphia
Karnath HO, Fruhmann Berger M, Kueker W, Rorden C (2004) The anatomy of spatial neglect based on voxelwise statistical analysis: a study of 140 patients. Cereb Cortex 14:1164-1172

Katzev M, Tüscher O, Hennig J, Weiller C, Kaller CP (2013) Revisiting the functional specialization of left inferior frontal gyrus in phonological and semantic fluency: the crucial role of task demands and individual ability. J Neurosci 33:7837-7845

Kimberg DY, Coslett HB, Schwartz MF (2007) Power in voxel-based lesion-symptom mapping. J Cogn Neurosci 19:1067-1080

Klein S, Staring M, Murphy K, Viergever MA, Pluim JP (2010) Elastix: a toolbox for intensity-based medical image registration. IEEE Trans Med Imaging 29:196-205

Kuijf HJ, Biesbroek JM, Viergever MA, Biessels GJ, Vincken KL (2013) Registration of brain CT images to an MRI template for the purpose of lesion-symptom mapping. Multimodal Brain Image Anal Lect Notes Comput Sci 8159:119-128

Levy DA, Bayley PJ, Squire LR (2004) The anatomy of semantic knowledge: medial vs. lateral temporal lobe. PNAS 101:6710-6715

Lezak MD, Howieson DB, Loring DW (2004) Neuropsychological assessment, 4th edn. Oxford University Press, New York

Loring DW, Meador KJ, Lee GP (1994) Effects of temporal lobectomy on generative fluency and other language functions. Arch Clin Neuropsychol 9:229-238

Magnusdottir S, Fillmore P, den Ouden DB, Hjaltason H, Rorden C, Kjartansson O et al (2013) Damage to left anterior temporal cortex predicts impairment of complex syntactic processing: a lesion-symptom mapping study. Hum Brain Mapp 34:2715-2723

Martin RC, Loring DW, Meador KJ, Lee GP (1990) The effects of lateralized temporal lobe dysfunction on formal and semantic word fluency. Neuropsychologia 28:823-829

Martin A, Wiggs CL, Lalonde F, Mark C (1994) Word retrieval to letter and semantic cues: a double dissociation in normal subjects using interference tasks. Neuropsychologia 32:1487-1494

Milner B (1964) Some effects of frontal lobectomy in man. In: Warren JM, Akert K (eds) The frontal granular cortex and behaviour. McGraw-Hill, New York, pp 313-334

Miotto EC, Bullock P, Polkey CE, Morris RG (1996) Spatial working memory and strategy formation in patients with frontal lobe excisions. Cortex 32:613-630

Molenberghs P, Sale MV (2011) Testing for spatial neglect with line bisection and target cancellation: are both tests really unrelated? PLoS One 6:e23017

Mummery CJ, Patterson K, Hodges JR, Wise RJ (1996) Generating 'tiger' as an animal name or a word beginning with T: differences in brain activation. Proc Biol Sci 22:989-995

Newcombe F (1969) Missile wounds of the brain. Oxford University Press, London

Nys GM, van Zandvoort MJ, de Kort PL, Jansen BP, van der Worp HB, Kappelle LJ et al (2005a) Domain-specific cognitive recovery after first-ever stroke: a follow-up study of 111 cases. J Int Neuropsychol Soc 11:795-806

Nys GM, van Zandvoort MJ, de Kort PL, van der Worp HB, Jansen $\mathrm{BP}$, Algra A et al (2005b) The prognostic value of domainspecific cognitive abilities in acute first-ever stroke. Neurology 64:821-827

Osterrieth PA (1944) Filetest de copied'une figure complex: contribution al'etude de la perception et de la memoire [The test of copying a complex figure: a contribution to the study of perception and memory]. Arch Psychologie 30:206-356

Perret E (1974) The left frontal lobe of man and the suppression of habitual responses in verbal categorical behaviour. Neuropsychologia 12:323-330

Rey A (1958) L'examin clinique en psychologie. Presses Universitaires de France, Paris 
Robinson G, Shallice T, Bozzali M, Cipolotti L (2012) The differing roles of the frontal cortex in fluency tests. Brain 135:2202-2214

Rorden C, Karnath HO (2004) Using human brain lesion to infer function: a relic from a past era in the fMRI age? Nat Rev Neurosci 5:813-819

Rorden C, Karnath HO, Bonilha L (2007) Improving lesion-symptom mapping. J Cogn Neurosci 19:1081-1088

Schwartz MF, Kimberg DY, Walker GM, Faseyitan O, Brecher A, Dell GS et al (2009) Anterior temporal involvement in semantic word retrieval: voxel-based lesion-symptom mapping evidence from aphasia. Brain 132:3411-3427

Theys C, De Nil L, Thijs V, van Wieringen A, Sunaert S (2013) A crucial role for the cortico-striato-cortical loop in the pathogenesis of stroke-related neurogenic stuttering. Hum Brain Mapp 34:2103-2112

Thothathiri M, Kimberg DY, Schwartz MF (2012) The neural basis of reversible sentence comprehension: evidence from voxel-based lesion-symptom mapping in aphasia. $\mathrm{J}$ Cogn Neurosci 24:212-222

Tulving E, Markowitsch HJ (1998) Episodic and declarative memory: role of the hippocampus. Hippocampus 8:190-204
Tzourio-Mazoyer N, Landeau B, Papathanassiou D, Crivello F, Etard O, Delcroix N et al (2002) Automated anatomical labeling of activations in SPM using a macroscopic anatomical parcellation of the MNI MRI single-subject brain. Neuroimage 15:273-289

Van Asselen M, Kessels RP, Neggers SF, Kappelle LJ, Frijns CJ, Postma A (2006) Brain areas involved in spatial working memory. Neuropsychologia 44:1185-1194

Van der Elst W, van Boxtel MP, van Breukelen GJ, Jolles J (2005) Rey's verbal learning test: normative data for 1855 healthy participants aged 24-81 years and the influence of age, sex, education, and mode of presentation. J Int Neuropsychol Soc 11:290-302

Verhage F (1964) Intelligence and age (in Dutch). Van Gorcum, Assen

Vossel S, Eschenbeck P, Weiss PH, Weidner R, Saliger J, Karbe H et al (2011) Visual extinction in relation to visuospatial neglect after right-hemispheric stroke: quantitative assessment and statistical lesion-symptom mapping. JNNP 82:862-868 